\section{Some aspects of computed tomography in Russia}

To the Editor: Computed tomography (CT) is increasingly used in Russia. Some CT departments are intensely busy, concentrating on outpatient cases. Free medical insurance covers only a small percentage of CT examinations, however; ${ }^{1}$ many patients pay for the procedure themselves, and some also prescribe it for themselves without consulting a physician, i.e. practice self-referral. ${ }^{2}$ Scanning is usually performed after following a standard protocol and takes several minutes; then the patient leaves and the next one is consulted. Detailed examination and analysis of images is performed by a radiologist in the late afternoon or evening, after the technicians have finished their work. Consequently, a radiologist's working day can last 14 hours or more, which can impair diagnostic quality because of fatigue. Such a workflow does not permit immediate re-examination with enhanced resolution or modified scanning parameters, which can be indicated for an unclear lesion or a group of lesions - for example, a pneumonia-like infiltrate or dissemination in a lung. ${ }^{3}$ One of the reasons for this workflow pattern is a shortage of upto-date literature and insufficient acquaintance with foreign experience. Misleading statements can be found in some domestic publications; for example: 'CT has become three-dimensional, which excludes missing small pathologic lesions and structures. ${ }^{5}$ Such generalisations tend to maintain a belief in the unlimited capabilities of 'computer diagnostics' in order, among other things, to enhance the number of self-referred patients. As a result, an expensive procedure accompanied by X-ray exposure is sometimes performed without justifiable indications.

Some excerpts from one of the most broadly used Russian manuals (translated verbatim): ' $\ldots$ uninterrupted scanning cycle of the spiral CT allows image reconstruction at any level ... In the spiral CT, a level of reconstruction does not depend on the main scanning parameters such as speed of the table or gantry positioning. As the scanning occurs uninterruptedly, the level of each section and the distance between adjacent sections are selected optionally by an operator not before but after the scanning ... Possibility of optional positioning of sections along the scanning axis and arbitrary choice of the section width allow forming blocks of partially overlapping images, whereas degree of mutual overlapping is practically unlimited ... In conventional CT, a similar effect can be achieved only if the table feed per gantry rotation would be less than the thickness of a tomographic layer ... In spiral CT, mutual overlapping of the slides is independent of the scanning parameters being a post-processing procedure ... location of pathological lesions between the tomographic layers, as it can occur in conventional CT, is therefore excluded. ${ }^{6}$

The impression is created by such assertions that a computer can reconstruct an image that is representative of the morphological substrate, on any level from the first to the last scanning plane. This notion does not take into account that a computer disposes only of the data that have been obtained as a result of radiodensity averaging of the tissue layers encompassed by the X-ray beam. If the pitch is $>1$, 'the patient would have a candy-striped appearance with unmarked flesh between ribbons of paint?

Another quotation (verbatim from the Russian): 'A result of the spiral scanning is an uninterrupted data volume, which can be arbitrarily subdivided into a required number of slides of optional thickness. ${ }^{6}$ Note that information flows uninterruptedly along a spiral line but not along the axis of table movement ( $\mathrm{z}$-axis), where information is intermittent and subdivided by intervals if the pitch is $>1$. A reconstruction algorithm interpolates the data from adjacent sections into the spaces between them. These spatial relationships can be illustrated by a plane cutting a helicoid. Overextension of the spiral, and excessive elevating of the pitch and/or collimation, can cause inadequate visualisation of pathological lesions.

It is sometimes argued that spatial resolution is defined only by pixel size (Tiurin IE, personal communication). One must note that pixel (or voxel) size is a characteristic of the equipment, while spatial resolution is defined also by information density per volume unit of the scanned tissue, which in turn depends on the scanning parameters. With high pitch values, the volume of interest is under-sampled. ${ }^{8}$ All the above applies also to the multi-slice spiral $\mathrm{CT}$, although spatial relationships here are more complicated: collimation values of a single section and of the whole detector array are distinguished, with 2 corresponding pitch values. Therefore, information density (as well as X-ray exposure) depends on the table feed per gantry rotation and on the distance between adjacent sections. The issue of radiation exposure in multi-slice spiral CT is beyond the scope of this letter, but the question should be posed in principle: Under which conditions is the enhanced X-ray exposure caused by crossings of beam trajectories with repeated exposure of the same tissue areas compensated by the advantages of multi-slice spiral CT, such as isotropy and high resolution volumetric data?

CT technology is progressing - scanning time is being reduced and image quality improved. This is no reason, however, to discard the principle of image analysis that is common to radiological and microscopic methods: in the case of an unclear lesion, one should be able to go to a 'high magnification' i.e. to repeat an examination with necessary adjustment to scanning parameters. It is therefore advantageous, when all images are examined by a radiologist, if the patient is still in the office, so that secondary scanning can be performed, if indicated. The total time per patient would probably be lower than in delayed image viewing, because a radiologist would not have to delve twice into a case. To maintain the same productivity, reception hours and, correspondingly, the working time of technicians should be prolonged. The great difference between the length of the working day for technicians and radiologists seems to be specific to Russia, as well as the relatively low remuneration for radiologists ( $<10 \%$ of the amount paid by the patient or insurer).

Additionally, it has been reported that CT productivity is enhanced when several technicians work simultaneously. ${ }^{10}$ The workflow described in the first paragraph (scanning performed after a standard protocol, and images viewed after reception hours) can be justified for screening purposes. However, the usefulness of CT screening and examination to selfreferred patients is questionable. ${ }^{2,11} \mathrm{~A}$ concluding point is that clinicians in modern hospitals have access to CT images via a computer network. ${ }^{12}$ But in many Russian hospitals, they can obtain only images on an Xray film and a verbal description. Overall, however, there are grounds for optimism: the improved economy now makes it possible to acquire modern equipment and literature, while broadening international cooperation will attract foreign expertise into the country.

\section{Sergei V Jargin}

Department of Pathology 
Peoples' Friendship University of Russia, Moscow

\author{
Elena E Jargina \\ Clinical Hospital Nr. 83, Moscow \\ sjargin@mail.ru
}

1. Jargin S. Some aspects of medical insurance in Russia. S Afr Med J 2008; 98(12): 907.

2. Fenton JJ, Deyo RA. Patient self-referral for radiologic screening tests: clinical and ethical concerns. J Am Board Fam Pract 2003; 16: 494-501.

3. Tiurin IE, Neishtadt AS, Sigina OA. Disseminated pulmonary tuberculosis: significance of high-resolution computerized tomography (in Russian) Vestn Rentgenol Radiol 1998; (6): 10-17.

4. Jargin SV. Limited access to foreign medical literature in Russia. CILIP Health Libraries Group Newsletter, 25(4). http://www.cilip.org.uk/specialinterestgroups/bysubject/health/newsletter (accessed 15 April 2009).

5. Ternovor SK, Sinitsyn VE. Progress in computed tomography and radiodiagnosis (in Russian with English summary) Ter Arkh 2006; 78(1): 10-12.

6. Tiurin IE. Computed Tomography of Thoracic Organs [in Russian]. St. Petersburg: ELBI, 2003: 72-73.

7. Cody DD, Mahesh M. AAPM/RSNA physics tutorial for residents: Technologic advances in multidetector CT with a focus on cardiac imaging. Radiographics 2007; 27: 1829-1837.

8. Prokop M. Principles of CT, spiral CT, and multislice CT. In: Prokop M, Galanski M, van der Molen AJ, Schaefer-Prokop C, eds. Spiral and Multislice Computed Tomography of the Body. Stuttgart: Thieme, 2003: 2-43.

9. Dalrymple NC, Prasad SR, El-Merhi FM, Chintapalli KN. Price of isotropy in multidetector CT. Radiographics 2007; 27: 49-62

10. Boland GW, Houghton MP, Marchione DG, McCormick W. Maximizing outpatient computed tomography productivity using multiple technologists. J Am Coll Radiol 2008; 5: 119-125.

11. Furtado CD, Aguirre DA, Sirlin CB, et al. Whole-body CT screening: spectrum of findings and recommendations in 1192 patients. Radiology 2005; 237: 385-394.

12. Van Ooijen PM, Bongaerts AH, Witkamp R, Wijker A, Tukker W, Oudkerk M. Multi-detector computed tomography and 3-dimensional imaging in a multi-vendor picture archiving and communications systems (PACS) environment. Acad Radiol 2004; 11: 649-660.

\section{Role of abdominal ultrasound in the context of the HIV/AIDS epidemic}

To the Editor: I read the article in the June 2009 edition of the SAJR entitled 'Trans-abdominal ultrasonic findings correlated with a CD4+ counts in adult HIV-infected patients in Benin, Nigeria, ${ }^{1}$ with interest.

I totally agree that the use of ultrasound (US) is invaluable in the assessment of HIV-positive patients and in the monitoring of treatment in these patients. I must, however, disagree with the statement that it should be used in all HIV-infected patients as a baseline investigation. The authors themselves clearly state that 'few of the ultrasound findings correlated statistically with the CD4+ counts.' This is unsurprising, given the unfocused use of US in terms of the patients' symptomatology in their study. The manpower needs to perform a baseline US study of the abdomen in every patient diagnosed with HIV, regardless of their clinical state, are also probably not realistically achievable, given the workload and staffing ratios of our hospitals.

In the appropriate clinical setting, however, US can be a vital tool in the assessment of patients with abdominal symptoms and in patients with an unexplained pyrexia in whom the chest X-ray is normal and sputum examination for acid-fast bacilli is negative. In this setting, US of the abdomen has the potential to detect many conditions, but in particular to demonstrate evidence of disseminated TB (which is a relatively common condition in our immuno-compromised population).

In 2002, Dr Mike Hunter and I published our experience with the use of abdominal sonar in HIV-infected patients with a fever of undetermined origin in a letter to the South African Medical Journal. ${ }^{2}$ Three findings were of particular note. The first was that pericardial effusion (which is easily demonstrated via the infra-sternal approach on abdominal US, and should be a routine component of every abdominal US) was a not uncommon, and often unexpected, finding, even in patients where the cardiac density was not enlarged on chest X-ray. Because small pericardial effusions are common in HIV-infected patients, we used a threshold of $5 \mathrm{~mm}$ for the diagnosis of a pathological collection. As tuberculous pericarditis is by far the most common cause of an effusion in this setting, patients with a pericardial effusion $>5 \mathrm{~mm}$ in width were started on treatment for TB and closely monitored.

Secondly: in our experience, the most common cause by far of abdominal lymphadenopathy in HIV-infected patients with pyrexia is tuberculous lymphadenitis. The distribution typically involves the porta hepatus, peripancreatic region, and occasionally the splenic hilum. The differential diagnosis includes lymphoma and persistent generalised lymphadenopathy (a component of the AIDS-related complex). Both these latter conditions appear to be very uncommon in our population. We therefore regard abdominal lymphadenopathy (in the appropriate clinical setting) as diagnostic of tuberculous lymphadenitis in the first instance, and therefore an indication for TB treatment. Should the patient fail to respond to antituberculous treatment (a very rare occurrence in our series), further investigation of the lymphadenopathy is indicated.

The third important finding in our series, in the diagnosis of disseminated TB, is that of splenic micro-abscesses. We found the demonstration of micro-abscesses within the spleen to be highly correlated with a diagnosis of disseminated TB. Although a number of other causes of splenic micro-abscesses (including salmonella, candida, lymphoma and histoplasmosis) have been described, disseminated TB is by far the most common cause in our population, and is regarded as an indication for commencing TB treatment. As with the other findings (as described above), these patients are closely monitored on both clinical and sonographic follow-up. Only patients who fail to respond to TB treatment (again, very rare in our series) are subjected to further investigation.

When used in this way, US of the abdomen results in substantial savings in terms of rendering more expensive and invasive investigations and their substantial costs unnecessary. This is of particular relevance in a resource-constrained setting, where access to sophisticated diagnostic modalities is limited. US, with its immediate results, also has the potential to save days (sometimes even weeks) in the time interval between performing the diagnostic examination and commencing treatment (compared with, for example, awaiting the results of tissue biopsy or bone marrow aspiration).

Although I do not dispute that the article is valuable in furthering our knowledge of HIV/AIDS via the medium of US imaging, I believe that there are very limited indications for US in asymptomatic patients, even those who are HIV-positive. However, in our hospital (and in several other institutions that I am aware of), abdominal US is not only being successfully used on a daily basis not only for the evaluation of patients with abdominal symptoms but also, according to the above criteria, in the diagnosis or exclusion of disseminated TB.

\section{J Emby}

AngloGold Ashanti Health, Western Deep Levels Hospital, Carletonville demby@anglogoldashanti.com 


\section{LETTERS}

1. Igbinedion B O-E, Marchie T T, Ogbeide E. Trans-abdominal ultrasonic findings correlated with CD4+ counts in adult HIV-infected patients in Benin, Nigeria. S Afr J Radiol 2009; 13(2): 34-40.

2. Emby D J, Hunter M. The value of ultrasound in the HIV-infected patient with a fever of undetermine origin. S Afr Med J 2002; 92(8): 566

Dr Igbinedion and co-authors reply: We thank Dr Emby for his interest in our article. During the course of the study, the recruiting physicians noted its usefulness, especially in renal scans, where we detected several asymptomatic nephropathies; consequently, these patients benefitted from improved follow-up laboratory reports after medical intervention. We are therefore of the opinion that radiologists should, if at all possible, not shy away from their responsibilities, even in the context of unbalanced staff ratios. This is one of the reasons why unqualified individuals offering ultrasonography in several Nigerian communities have led to increased frequencies of quackery and missed diagnosis, which is a major and potentially disastrous non-biological hazard of ultrasound.
Our study was rather an abridged version of a broader perspective. In the study, fever, cough, skin rash and diarrhoea were the most common presenting complaints (45.3\%, 25\%, 20\% and $14.7 \%$ respectively) while uncommon complaints constituted only $8.6 \%$. Unfortunately, apart from skin rash, these symptoms did not correlate significantly with abnormal sonographic findings on univariate analysis. But, as would be expected, patients with abdominal symptoms had more abnormal sonographic findings compared with those with other complaint or no presenting complaint at all. However, since we are currently undertaking a followup study, there may be further interesting findings in the offing.

\section{B O-E Igbinedion}

Department of Radiology, College of Medical Sciences, University of Benin and University of Benin Teaching Hospital, Benin, Edo, Nigeria igbins2@yahoo.com 